\title{
Comparação da Frequência de Queixa de Vertigem no Idoso Com e Sem Hipertensão Arterial
}

\section{Comparison of Frequency of Vertigo in Elderly with And without Arterial Hypertension}

\author{
Luciana Lozza de Moraes Marchiori*, Juliana Jandre Melo**, Fernanda Luisa de Figueiredo Possette ${ }^{* * *}$ \\ Ariane Leme Correa $* * * *$. \\ * Doutorado em Medicina e Ciências da Saúde - UEL. Professor Pesquisador. \\ *** Mestrado em Fonoaudiologia. Professor Adjunto I. \\ *** Graduação em Fonoaudiologia. Bolsista de iniciação científica do Curso de Fonoaudiologia da UNOPAR, Londrina, Paraná, Brasil no ano de 2009. Fonoaudióloga Clínica. \\ ***** Graduação em Fonoaudiologia. Fonoaudióloga Clínica. \\ Instituição: Universidade Norte do Paraná - UNOPAR. \\ Londrina / PR - Brasil. \\ Endereço para correspondência: Luciana Lozza de Moraes Marchiori - Campus Universitário de Londrina - Clinica de Fonoaudiologia - Avenida Paris, 675 - Jardim Piza \\ - Londrina / PR - Brasil - CEP: 86041-140 - Caixa Postal: 401 - Telefone: (+55 43) 3371-7775 - E-mail: luciana.marchiori@unopar.br \\ Artigo recebido em 6 de Agosto de 2010. Artigo aprovado em 4 de Setembro de 2010.
}

\section{RESUMO}

Introdução:

A presença de sintomas otológicos associados à hipertensão é discutida há décadas; entretanto, sua etiologia ainda permanece obscura.

Objetivo:

Método:

Comparar a frequência de queixa de vertigem em pacientes idosos com e sem hipertensão arterial.

Estudo prospectivo com 238 indivíduos. Foi aplicado um questionário para preenchimento sistematizado de dados para verificação da hipertensão arterial e da vertigem baseado no protocolo para anamnese audiológica utilizada na rotina de atendimentos.

Resultados e Discussão: Houve uma proporção maior de mulheres em ambos os grupos. Quanto à idade, houve média de idade semelhante entre o grupo entre os dois grupos. Quanto ao uso de medicamentos, todos faziam uso de algum tipo de medicamento de forma continua. Na população estudada com hipertensão arterial, 106 (90\%) faziam uso de anti-hipertensivos. Dos 99 indivíduos que relataram vertigem 22 (15,15\%) dos idosos faziam uso de antivertiginosos. No grupo de hipertensos 58 (23,8\%) indivíduos relataram vertigem e no de não hipertensos 41 (16\%) indivíduos relataram vertigem. Não houve significância entre a queixa de vertigem e a hipertensão arterial no grupo estudado, isto provavelmente por alguns critérios como a exclusão de indivíduos com enfermidades e uso de medicamentos capazes de produzir vertigem, que não puderam ser evitados durante a execução, pois a maioria deles tinha distúrbios associados, situação comum nos idosos.

Conclusão: Observou-se em idosos, com e sem hipertensão arterial, grande frequência da queixa de vertigem, embora

Palavras-chave: não se tenha obtido relação significativa entre a queixa de vertigem e hipertensão arterial na população estudada.

\section{SUMMARY}

Introduction: hipertensão, vertigem, saúde do idoso.

Objective:

Method:

The otologic symptoms associated with hypertension are discussed for decades, yet its etiology remains obscure.

To compare the frequency of vertigo in elderly patients with and without hypertension.

A prospective study of 238 individuals. We administered a questionnaire to fill in systematic data for verification of hypertension and vertigo-based protocol for audiological anamnesis used in routine care.

Results and Discussion: There were a higher proportion of women in both groups. As for age, mean age was similar between the groups between the two groups. Regarding the use of medication, all were using some kind of medication continuously. In the study population with hypertension, 106 (90\%) were using antihypertensive drugs. From 99 individuals who reported dizziness 22 (15.15\%) of seniors were using anti-vertigo. Hypertensive patients $58(23.8 \%)$ subjects reported dizziness and 41 non-hypertensive (16\%) subjects reported dizziness. There was no significance between vertigo and hypertension in the study group, this probably by some criteria as to exclude individuals with diseases and use of medications capable of producing dizziness, which could not be avoided during the implementation, since most of them had associated disorders, common in the elderly.

Conclusion: It was observed in the elderly with and without hypertension, high frequency of vertigo, although we have not achieved significant relationship between vertigo and hypertension in this population.

Keywords: hypertension, vertigo, aging health. 


\section{INTRODUÇÃO}

Uma vez que a expectativa de vida da população mundial vem crescendo, inúmeros estudos têm sido desenvolvidos com intuito de contribuir para a melhoria da qualidade de vida na terceira idade.

Entre estes estudos estão os relacionados à hipertensão arterial, devido a sua grande prevalência nesta população.

Como a hipertensão arterial pode causar alterações vestibulares em decorrência do comprometimento periférico e/ou central do sistema vestibular, ocasionando vertigem, uma parcela dos estudos relacionados à terceira idade tem sido realizado visando verificar tanto a relação entre a hipertensão arterial e a vertigem, como as atitudes a serem tomadas para minimizar ou sanar este sintoma.

Existe disfunção do equilíbrio denominada tontura, sempre que há conflito na integração de informações sensoriais responsáveis pelo controle postural prejudicando a sustentação do indivíduo no espaço (1). A tontura que, quando rotatória, é denominada vertigem, é uma sensação de perturbação do equilíbrio, presente em uma infinidade de doenças, atingindo principalmente idosos. Sua origem está correlacionada em 85\% dos casos com distúrbios do sistema vestibular, ocorrendo a sua sintomatologia em geral, durante a movimentação da cabeça ou mudanças posturais $(2,3)$.

A grande incidência de distúrbios labirínticos se deve em parte à hipersensibilidade do labirinto a vários distúrbios como: hormonais, metabólicos, cervicais e circulatórios. Estudos mostram que alterações auditivas e vestibulares podem ser secundárias à hipertensão arterial sistêmica $(3,4)$.

Todas as células vivas precisam de fornecimento adequado de oxigênio e nutrientes para manterem suas funções de maneira adequada, este fornecimento depende da integridade funcional e estrutural do coração e dos vasos sanguíneos (5). A hipertensão arterial pode facilitar alterações estruturais do coração e vasos sanguíneos (6).

O comprometimento do aparelho circulatório pode prejudicar o ouvido interno sendo que, um dos mecanismos fisiopatológicos descritos é o aumento da viscosidade sanguínea, que acarreta uma diminuição do fluxo sanguíneo capilar e consequentemente o transporte de oxigênio (7).

A partir destas colocações resolveu-se neste trabalho comparar a frequência de queixa de vertigem em pacientes idosos com e sem hipertensão arterial.

\section{MÉTODO}

A pesquisa foi realizada através de estudo com delineamento transversal, após a análise e aprovação do projeto, PP/0063/09, bem como do termo de consentimento pela resolução 196/96-CNS.

Foram incluídos 238 indivíduos, com idade acima de 60 anos. A população foi selecionada na sequência dos atendimentos da Clínica de Fonoaudiologia da Universidade Norte do Paraná entre os anos de 2008 e 2009.

Foram excluídos deste estudo, pacientes com história alterações metabólicas, como diabetes e de distúrbios vasculares como acidente vascular cerebral. Também foram excluídos indivíduos portadores de insuficiência renal crônica.

Os pacientes foram entrevistados pelas alunas voluntárias e bolsistas de iniciação científica supervisionadas pelas fonoaudiólogas responsáveis pela pesquisa.

Foi aplicado um questionário para preenchimento sistematizado de dados clínicos tais como nome, idade, gênero e as seguintes perguntas: o senhor tem pressão alta? Tem medido sua pressão arterial ultimamente? Quando mediu a pressão pela última vez? Quanto tem dado sua pressão arterial? O nome do médico? Ou a Unidade de Saúde onde faz controle? Toma remédio para a pressão arterial, qual ou quais os medicamentos que toma?. Foram respeitadas as normas estabelecidas pelo III Consenso Brasileiro de Hipertensão Arterial de 1998. Sendo considerados portadores de hipertensão arterial àqueles que responderam afirmativamente as perguntas referentes à patologia e identificaram o(s) medicamento(s) que tomavam. Foi realizada anamnese com dados clínicos para verificação da vertigem baseado no protocolo utilizado na rotina de atendimentos da clinica escola.

A associação entre a queixa de vertigem e hipertensão arterial foi analisada mediante as estimativas da odds ratios (OR), por ponto e por intervalo e do valor da estatística pelo Teste Qui-quadrado de MantelHaenszel.

\section{RESULTADOS}

Houve uma proporção maior de mulheres em ambos os grupos, sendo $123(51,68 \%)$ da população do gênero feminino e 115 (48,31\%) do gênero masculino. Quanto à idade, houve uma média de idade semelhante entre o grupo com hipertensão arterial e o grupo sem hipertensão arterial. 
Quanto ao uso de medicamentos, todos os indivíduos faziam uso de algum tipo de medicamento de forma continua como anti-hipertensivos, antiinflamatórios nãoesteroidais, fitoterápicos, diuréticos, vasodilatadores, antiarrítmicos, cardiotônicos, benzodiazepínicos, hipoglicemiantes orais, antidepressivos antiulcerosos e complexos vitamínicos. Os anti-hipertensivos foram os medicamentos mais citados, seguidos dos diuréticos.

Na população estudada com hipertensão arterial, 106 (90\%) faziam uso de anti-hipertensivos na data da aplicação dos questionários, prevalecendo o uso de betabloqueadores, seguidos dos antagonistas de receptor da angiotensina II, dos bloqueadores do canal de cálcio e dos alfa-bloqueadores. Destes pacientes, que utilizavam medicamentos, 22 (21\%) indivíduos faziam uso de mais de um fármaco anti-hipertensivo, sendo a associação entre diuréticos e inibidores de enzima conversora de angiotensina, a mais referida.

Dos 99 indivíduos que relataram vertigem, 15 $(15,15 \%)$ indivíduos faziam uso de antivertiginosos na época da pesquisa, prevalecendo o uso de dicloridrato de flunarizina.

Não houve significância entre a queixa de vertigem e a hipertensão arterial no grupo estudado (Tabela 1).

\section{DISCUSSÃO}

A hipertensão arterial e a insuficiência cardíaca podem causar alterações auditivas e vestibulares em decorrência do comprometimento periférico e/ ou central dos sistemas auditivo e/ou vestibular, além do uso de medicamentos para alterações metabólicas e circulatórias, relatados pelos pacientes, também podem afetar a orelha interna e provocar tontura $(8,9,10,11)$. Assim, pacientes que apresentam distúrbios associados (situação comum nos idosos) que potencialmente podem causar tontura, terão uma prevalência maior de vertigem e um prejuízo ainda maior da qualidade de vida. Isto se pode observar neste estudo, que apesar de não mostrar associação entre hipertensão arterial e vertigem nestes idosos, verificou que há grande frequência tanto de vertigem como de hipertensão arterial nesta população.

No que se refere à metodologia deste estudo, apesar do cuidado na delimitação da idade, concentrando a faixa etária em indivíduos acima de 60 anos, alguns critérios como a exclusão de indivíduos com enfermidades e uso de medicamentos capazes de produzir vertigem certamente, não puderam ser evitados durante a execução, uma vez que a grande maioria dos pacientes apresentava distúrbios associados, situação comum nos idosos, que
Tabela I. Distribuição completa do número de pacientes segundo hipertensão arterial e queixa de vertigem.

\begin{tabular}{|c|c|c|c|c|c|}
\hline \multirow{3}{*}{$\begin{array}{l}\text { Hipertensão } \\
\text { Arterial }\end{array}$} & \multicolumn{4}{|c|}{ Vertigem } & \multirow[t]{3}{*}{ TOTAL } \\
\hline & \multicolumn{2}{|c|}{$\operatorname{Sim}$} & \multicolumn{2}{|c|}{ Não } & \\
\hline & $N$ & $\%$ & $N$ & $\%$ & \\
\hline Sim & 58 & $23,8 \%$ & 60 & $27,0 \%$ & 118 \\
\hline Não & 41 & $16,8 \%$ & 79 & $32,4 \%$ & 120 \\
\hline$\overline{\text { TOTAL }}$ & 99 & & 139 & & 238 \\
\hline
\end{tabular}

$\chi_{\text {corr }}^{2}=3.514(p=0.0608)$

Termo de Consentimento Livre Esclarecido

Nome do participante:

Data:

Responsável: Profa Luciana Lozza de Moraes Marchiori

Colaboradora: Juliana Jandre Melo

Discente:

Docentes e discente da Universidade Norte do Paraná UNOPAR - Avenida Paris, 675 - Jardim Piza - (43) 337। -7700 - CEP 8604I - 100 - Londrina - Paraná.

"COMPARAÇÃO DA FREQUÊNCIA DE QUEIXA DE VERTIGEM NO IDOSO COM E SEM HIPERTENSÃO ARTERIAL"

O propósito deste estudo é verificar a associação de Hipertensão Arterial com VPPB (vertigem pocisional paroxística benigna) em indivíduos a partir de 60 anos de idade.

Serão empregadas duas etapas para a realização desta pesquisa: assinatura do consentimento livre esclarecido e aplicação de questionário padrão do projeto de vertigem e perguntas referentes a Hipertensão Arterial.

Os procedimentos utilizados são simples, rápidos e indolores ao participante. Não existem riscos ou desconforto associados a ele ou afamília. Estes procedimentos realizados serão gratuitos.

Os dados colhidos são sigilosos e só serão utilizados na pesquisa mediante a autorização do mesmo, que poderá se retirar da pesquisa em qualquer momento.

Qualquer dúvida, entrar em contato na Clinica dos Distúrbios da Comunicação Humana da UNOPAR através do número (43) 337 I-7775 a qualquer momento.

\section{Consentimento Pós Esclarecido}

Declaro que, depois de convencido pelo pesquisador e ter entendido o que me foi explicado, aceito participar da presente pesquisa.

Londrina, de de 200

Assinatura do participante (responsável)

Assinatura do docente responsável

Assinatura do discente 
potencialmente por poderem ocasionar vertigem, podem ter ocasionado vieses, que refletiram na não significância entre as variáveis de estudo.

Cada vez mais se tem abordado o envelhecimento populacional, em todas as áreas de estudo principalmente na de saúde, isto porque ele se constitui atualmente em uma realidade mundial. No Brasil, o número absoluto de pessoas com mais de 60 anos aumentou nove vezes nas últimas seis décadas. Em 1940 era de 1,7 milhões e em 2000 saltou para 14,5 milhões, projetando-se para 2020 um contingente de aproximadamente 30,9 milhões de pessoas com mais de 60 anos (12).

Tendo-se em vista a expectativa de vida cada vez mais alta, vários estudos têm sido desenvolvidos de modo a contribuir para a melhoria da qualidade de vida na terceira idade, sendo consideradas as magnitudes e as diferenças de cada grupo sobre o que eles mesmos valorizam na busca de seu bem-estar, além de verificarem seus hábitos e cuidados com a saúde, incluindo abordagens sobre o uso de medicamentos, doenças crônicas e atividades físicas $(13,14,15,16)$.

Salienta-se a grande porcentagem de queixa de vertigem, tanto no grupo de hipertensos $23,8 \%$ como no grupo de não hipertensos 16\%, que vem de encontro com a literatura da área sobre o assunto17,18 com intuito de citar que atitudes para verificar e tratar a vertigem em idosos certamente devem ser tomadas. Tais atitudes certamente ajudarão os muitos pacientes que deliberadamente restringem as atividades físicas, viagens e reuniões sociais, com a intenção de reduzir o risco de aparecimento destes sintomas desagradáveis e assustadores, e para evitar o embaraço social e o estigma que eles podem causar $(15,16,17,18)$.

Há também necessidade de que todos os profissionais da área de saúde, principalmente aqueles envolvidos com idosos, tenham conhecimentos tanto sobre a etiologia e sintomatologia da vertigem, como sobre o tratamento e consequências da mesma, informando a população sobre a vertigem de modo geral e mais especificamente sobre as atitudes a serem tomadas frente a um quadro de crise vertiginosa $(19,20,21,22)$.

\section{CONCLUSÃo}

Observou-se nesta pesquisa em idosos, com e sem hipertensão arterial, grande frequência da queixa de vertigem, embora não se tenha obtido relação significativa entre a queixa de vertigem e hipertensão arterial na população estudada. Isto serve de base para que esse sintoma deva ser investigado e tratado não apenas na população de hipertensão, mas em idosos de modo geral, visando melhoria na qualidade de vida desta população.

\section{REFERENCIAS BIBLIOGRÁFICAS}

1. Ganança FF, Castro ASO, Branco FC, NatourJ. Interferência da tontura na qualidade de vida de pacientes com síndrome vestibular periférica. Rev Bras Otorrinolaringol. 2004, 70(1):97-101.

2. Ganança MM, Caovilla HH, Munhoz MSL, Silva MLG. Alterações da audição e do equilîbrio corporal no idoso. Rev Bras Med. 1999, 56(10):995-1011.

3. Teixeira LJ, MachadoJNP. Manobras para o tratamento da vertigem posicional paroxística benigna: revisão sistemática da literatura. Rev Bras Otorrinolaringol. 2006, 72(1):130-9.

4. Mor R, Fragoso M, Figueiredo JF, Taguchi CK. Vestibulometria e fonoaudiologia. São Paulo: Lovise; 2001.

5. Maia RA, Diniz FL, Carlesse A. Manobras de reposicionamento no tratamento da vertigem paroxística posicional benigna. Rev Bras Otorrinolaringol. 2001, 67(5):612-6.

6. Marková M. The cochleovestibular syndrome in hypertension. Cesk Otolaryngol. 1990, 39(2):89-97.

7. Bachor E, Selig YK, Jahnke K, Rettinger G, Karmody CS. Vascular variations of the inner ear. Acta Otolaryngol. 2001, 121(1):35-41.

8. Katz, J. Tratado de audiologia clínica. São Paulo: Manole; 1989.

9. Murray KJ, Hill K, Phillips B, Waterston J. A pilot study of falls risk and vestibular dysfunction in older fallers presenting to hospital emergency departments. Disabil Rehabil. 2005, 2(9):499-506.

10. Tiensoli LO, Couto ER, Mitre EI. Fatores associados à vertigem ou tontura em indivíduos com exame vestibular normal. Rev CEFAC. 2004, 6(1):94-100.

11. Marchiori LLM, Rego Filho EA, Matsuo T. Hipertensão como fator associado à perda auditiva. Rev Bras Otorrinolaringol. 2006, 72(4):533-40.

12. Santos MRDR. Caracterização nutricional de idosos com hipertensão arterial em Teresina-PI. Rev. Bras. Geriatr. Gerontol. 2007, 10(1):1-11.

13. Vecchia R, Riuz T, Bocchi S, Corrente J. Qualidade de 
vida na terceira idade: um conceito subjetivo. Rev Bras Epidemiol. 2005, 8(3):246-53.

14. Marchiori LLM, Zumbido ehipertensãoarterial no processo de envelhecimento. Revista Brasileira de Hipertensão. 2009, 16(1):5-8.

15. Ganança FF, Castro ASO, Branco FC, Natour J. Interferência da tontura na qualidade de vida de pacientes com síndrome vestibular periférica. Rev Bras Otorrinolaringol. 2004, 70(1):97-104.

16. Levandowski A, Bueno VK, Marchiori LLM, Melo JJ. Vertigem no idoso: relato de caso. Rev. CEFAC. 2008, 10(4):588-591.

17. Marchiori LLM Rego Filho EA. Queixa de vertigem e hipertensão arterial. Rev. CEFAC. 2007, 9(1):116-121.

18. Chung KW, et al. Incidence of horizontal canal benign paroxysmal positional vertigo as a function of the duration of symptoms. Otol Neurotol. 2009, 30(2):202-5.

19. Marchiori, L.L.M. Zumbido e hipertensão arterial no processo de envelhecimento. Rev Bras Hiperten. 2009, 16:5-8.

20. Judd O. What do patients understand by the term "Vertigo": a cross-sectional study. Clin Otolaryngol. 2009, $54: 4$.

21. Jolobe O. Potential causes of delayed diagnosis include convulsive syncope and cardiogenic vertigo. QJ Med. 2009.

22. Zingler VC, et al. Causative factors, epidemiology, and follow-up of bilateral vestibulopathy Ann NY Acad Sci. 2009, 1:505-8.

22. Warninghoff JC, et al. Co-morbidities of vertiginous diseases. BMC Neurol. 2009, 9(1):29. 